\title{
Development of Fourier-transformed mid-infrared spectroscopy prediction models for major constituents of fractions of delactosated, defatted milk obtained through ultra- and nanofiltration
}

\author{
Marco Franzoi, ${ }^{* 1}$ Carmen L. Manuelian, ${ }^{*}$ Luigi Rovigatti, $†$ Emanuela Donati, $\dagger$ and Massimo De Marchi* \\ *Department of Agronomy, Food, Natural Resources, Animals and Environment, University of Padova, Viale dell'Università 16, \\ 35020 Legnaro (PD), Italy \\ †Granarolo SpA, Via Cadriano 27/2, Bologna, Italy
}

\begin{abstract}
Milk filtration procedures are gaining relevance in the dairy industry because milk ultra- and nanofiltrates are used to increase milk processing efficiency, and as additives for products with improved nutraceutical properties. This study aimed to develop Fourier-transformed mid-infrared spectroscopy calibrations for ultra- and nanopermeate and retentate fractions of defatted and delactosated milk. A total of 154 samples from different milk fractions were collected and analyzed using reference methods to determine protein, solids-not-fat, glucose, and galactose content. The obtained values were matched with their respective Fourier-transformed mid-infrared spectroscopy spectra to develop new prediction models. Calibrations for each trait were built following 3 different approaches to get the best prediction models: (1) using the entire data set, (2) using 3 subsets based on component concentrations (level approach), and (3) using hierarchical clusters calculated with pairwise Mahalanobis distance among spectra (cluster approach). Calibrations were developed using partial least squares regression, after removing low signal-to-noise ratio wavelengths, and validated through a leave-one-out cross-validation procedure. In addition, the accuracy of the predicted values within each fraction was checked for each approach. Dividing the data set into subsets improved prediction models for each trait and for the samples in each milk fraction. Without considering milk fraction, the best improvement was observed for glucose and galactose. Glucose ratio performance deviation in cross-validation (RPD) increased from 7.42 to 11.31 and 11.06, for cluster and level approaches, respectively, whereas galactose RPD increased from 8.86 to 11.69 and 11.27 for cluster and
\end{abstract}

Received December 24, 2017.

Accepted April 3, 2018.

${ }^{1}$ Corresponding author: marco.franzoi@studenti.unipd.it level approaches, respectively. Considering milk fractions, the best improvement was observed for protein content, where RPD ranged from 0.08 to 6.06 for the whole data set calibration, whereas it ranged from 0.43 to 40.34 for the subset calibration approaches. Cluster and level approaches to build calibration models were comparable for samples from different fractions, suggesting that the 2 subsetting protocols should be both investigated to get the best prediction performances.

Key words: filtration, clustering, glucose, galactose

\section{INTRODUCTION}

Milk ultra- and nanofiltration are common stages in dairy processes, applied both for milk concentration and for the recovery of nutritional constituents from milk and whey (Khatkar et al., 2014; Sturaro et al., 2014; Agarwal et al., 2015; Moreno-Montoro et al., 2015). Actually, filtration procedures are preferred for the recovery of expensive or heat-sensitive components as an alternative to unit operations such as centrifugation and evaporation (Pouliot, 2008). Moreover, filtration procedures allow the development of new products and valorization of dairy industry side-products (Kumar et al., 2013). The most common filtration procedures are based on ceramic or polymeric membranes with different pore sizes, where permeate is separated from retentate applying a defined pressure to the system (Kumar et al., 2013). Based on nominal pore size of membranes, procedures can be divided into (1) microfiltration, for the separation of cells, fat globules, and bacteria; (2) UF, with most proteins found in the retentate; (3) nanofiltration, which is selective for molecules from sugars to salts; and (4) reverse osmosis, which is able to decrease water content of the product (Brans et al., 2004). Considering the increasing market for products derived from milk filtration, the validation of on-line and at-line methods for the evaluation of retentate and permeate composition is essential to develop their industrial applications. 
Fourier-transformed mid-infrared spectroscopy (FTMIR) analyses are a cost-effective method for at-line analysis of dairy products (Goulden, 1956; De Marchi et al., 2014; Wang et al., 2017). Moreover, FT-MIR has demonstrated its effectiveness in predicting milk technological traits, in addition to its composition (De Marchi et al., 2014; Visentin et al., 2016, 2017; McDermott et al., 2017). Nevertheless, the development of new prediction models is required when new products or new traits need to be analyzed. Few data are available regarding infrared applications for monitoring ultra- and nanofiltration processes (Barbano and Dellavalle, 1987; Solís-Oba et al., 2011). The predictability of FT-MIR calibration is strongly influenced by the number and variability of the reference data and the quality of the recorded spectra (Naes et al., 2002). Moreover, inclusion in the prediction model of sources of variability, such as the use of different standardized instruments, demonstrated an increase in calibration reliability (McKenna, 2001; Agelet and Hurburgh, 2010). This paper aimed to develop and compare 3 different FT-MIR prediction methods to determine glucose, galactose, protein, and SNF content in products derived from subsequent filtrations of defatted, delactosated milk.

\section{MATERIALS AND METHODS}

\section{Sample Collection}

Fractionation of bulk defatted milk (DFM) is routinely done by Granarolo S.p.A. (Bologna, Italy), which is the largest Italian dairy company (Benedet et al., 2018), at industrial scale following internal procedures to concentrate milk and to recover nutritional constituents from milk and whey. Briefly, bulk DFM is delactosated (DLM) and thereafter ultra- and nanofiltrated. Two fractions are obtained by UF of DLM: permeate (PUF) and retentate (RUF) of DLM. The PUF fraction is subsequently nanofiltered with a polymeric membrane, again obtaining a retentate and a permeate named RN1 and PN1, respectively. The PN1 fraction is subjected to a final nanofiltration step with a polymeric membrane at very low porosity obtaining the 2 final fractions named PN2 and RN2 for the new permeate and retentate, respectively. A total of 154 samples of starting milk or milk fractions were collected in Granarolo S.p.A. Each sample differentiates for fractionation step, starting bulk milk or fraction, or process parameters such as pressure and temperature. For each sample, 3 aliquots were collected: one was immediately frozen and kept at $-20^{\circ} \mathrm{C}$ for reference analysis, and the other 2 aliquots were used for FT-MIR spectra collection.

\section{Infrared Spectra Acquisition}

Each sample was analyzed using 2 FT-MIR instruments, Milkoscan FT2 and Milkoscan FT1 (Foss Electric A/S, Hillerød, Denmark) located at Granarolo S.p.A. facilities in Granarolo (Bologna, Italy) and Gioia del Colle (Bari, Italy), respectively, routinely standardized accordingly to the manufacturer. The optical standardization of the instruments, along with the standardization of the sample preparation and presentation, permits to merge in the same database spectra obtained from different instruments to develop a more robust calibration that will work on both instruments adjusting only the slope and the bias (McKenna, 2001). In the case that more than one spectrum from the same sample and same instrument was recorded, only one was randomly selected. Thus, 2 spectra were associated with each reference data point, one from each instrument. Before doing the reference analysis, spectral outliers were checked within each milk fraction using Mahalanobis distance, detecting 5 spectral outliers. The 2 spectra corresponding to the samples of the spectral outliers were removed from the data set. The final data set included 298 spectra from 149 samples. Spectra were recorded at room temperature within $24 \mathrm{~h}$ of sample collection. Spectral information of each sample contained 1,060 data points in the region between 5,000 and $900 \mathrm{~cm}^{-1}$ and was recorded as $\log (1 /$ transmittance $)$.

\section{Reference Analysis}

Ultrapure laboratory-grade (mQ) water was produced with Arium 611 UV (Sartorius, Monza Brianza, Italy), and all chemicals were bought from SigmaAldrich (Saint Louis, MO), unless otherwise indicated, at the highest available purity.

The 149 samples were analyzed for glucose and galactose content by HPLC, partially modifying the method proposed by Indyk et al. (1996). One milliliter of homogenized sample was diluted in $8.5 \mathrm{~mL}$ of $\mathrm{mQ}$ water, briefly mixed, and then $0.50 \mathrm{~mL}$ of Carrez 1 solution $\left\{3.60 \mathrm{~g}\right.$ of $\mathrm{K}_{4}\left[\mathrm{Fe}(\mathrm{CN})_{6}\right] \cdot 3 \mathrm{H}_{2} \mathrm{O}$ in $100 \mathrm{~mL}$ of $\mathrm{mQ}$ water $\}$ was added. After mixing, $0.50 \mathrm{~mL}$ of Carrez 2 solution $\left(7.20 \mathrm{~g}\right.$ of $\mathrm{ZnSO}_{4} \cdot 7 \mathrm{H}_{2} \mathrm{O}$ in $100 \mathrm{~mL}$ of $\mathrm{mQ}$ water) was added and immediately vortexed for $10 \mathrm{~s}$. Thereafter, the preparation was incubated at room temperature for $30 \mathrm{~min}$ and centrifuged at $8,000 \times g$ for $10 \mathrm{~min}$ at room temperature. The obtained supernatant was diluted $1: 10$ and passed through a $0.45 \mu \mathrm{m}$ filter. Finally, $10 \mu \mathrm{L}$ of the resulting sample was injected in a HPLC Spectra system (Thermo Finnigan, Waltham, MA) equipped with Aminex HPX 87C column (Bio-Rad, Hercules, 
CA) with temperature set at $80^{\circ} \mathrm{C}, \mathrm{mQ}$ water as mobile phase with flux set at $0.6 \mathrm{~mL} / \mathrm{min}$, and carbohydrates signals obtained using a Waters (Sesto San Giovanni, Italy) 410 refractometer.

Protein content and SNF was also analyzed in 112 of the samples according to official methods (AOAC International, 2000). After mineralization of the sample, the Kjeldahl method by Kjeltec 2300 Foss Tecator (Foss Electric A/S) was used for protein content determination, whereas SNF was determined by exsiccation at $100^{\circ} \mathrm{C}$ for $4 \mathrm{~h}$ (AOAC International, 2000).

\section{Statistical Analysis}

Reference values within each fraction were checked for normality according to Shapiro-Wilk test before statistics calculation. For each major constituent (protein, SNF, glucose, and galactose), only spectra from the samples analyzed with the reference method were retained. That means 298 spectra for glucose and galactose, and 224 spectra for protein and SNF. Due to the limited number of samples obtained for each milk fraction, and the need to obtain reliable calibrations for each fractionation stage, we explored the possibility to group different fractions to obtain single calibrations. Calibrations were built following 3 different approaches to get the best prediction models: (1) using the entire data set, (2) using 3 subsets based on component concentrations (level approach), and (3) using 3 hierarchical clusters calculated with pairwise Mahalanobis distance among spectra (cluster approach). The accuracy of the predicted values obtained with those approaches was checked within each milk fraction.

Based on component concentration, samples were distributed in 3 groups for each trait. Samples were grouped taking into account the reference data distribution. For glucose and galactose group 1 included samples with concentration $<3 \%$, group 2 included samples with concentration ranging between 3 and $8 \%$, and group 3 included samples with concentration $>8 \%$. For protein, groups 1, 2, and 3 included samples with concentration $<2.5 \%, 2.5-4 \%$, and $>4 \%$, respectively. For SNF, samples were grouped as 1, 2, and 3 for concentration $<12 \%, 12-22 \%$, and $>22 \%$, respectively.

Based on spectral data, samples were clustered using SAS ver. 9.4 (SAS Institute Inc., Cary, NC) calculating pairwise Mahalonobis distance. Ten principal components were calculated using PROC PRINCOMP of SAS. Pairwise Euclidean distances were calculated using as input weighted principal components. The obtained distance matrix was used as input for PROC CLUSTER of SAS, imposing centroid method and 3 final clusters, resulting in 0.73 of variance ratio (Sokal and Michener, 1958).

Prediction models for the complete data set, and for each individual level and cluster were developed using partial least squares (PLS) regression in SAS ver. 9.4 with spectral range from 964.5 to $1,581.78 \mathrm{~cm}^{-1}$, from $1,716.81$ to $2,295.51 \mathrm{~cm}^{-1}$, and from $2,411.25$ to $2,970.66 \mathrm{~cm}^{-1}$, to exclude low signal-to-noise ratio regions according to the FT-MIR instrument producer, for a final set of 338 data points for each spectrum. Prediction equations were validated using a leaveone-out cross-validation, excluding a single sample $(2$ spectra) from each iteration. The optimal number of factors for each calibration model was selected based on the lowest predicted residual sum of squares (Allen, 1974). The goodness-of-fit statistics considered were the coefficient of determination in calibration and in cross-validation $\left(\mathbf{R}^{2}\right.$ and $\mathbf{R}_{\mathbf{C V}}^{2}$, respectively); standard error of calibration (SEC); standard error of prediction in cross-validation (SECV); and ratio performance deviation in cross-validation (RPD), calculated as the ratio between standard deviation (SD) and SECV (Williams and Sobering, 1993). To compare all 3 methods, for the level and the cluster approaches the goodness-of-fit statistics were recalculated, joining the predicted values estimated for each level or cluster, from the corresponding calibration. Hence, we had one goodness-of-fit statistics output for each approach (i.e., complete data set, level, and cluster). In addition, the goodness-of-fit statistics for each fraction were also calculated considering the predicted values obtained with each approach.

\section{RESULTS AND DISCUSSION}

\section{Milk Filtrated Fraction Composition}

Average values for each major constituent and fractionation group are displayed in Table 1. Ultrafiltration retained about $95 \%$ of proteins, whereas sugars and salts were free to permeate (Table 1; Figure 1). A slight difference in sugar concentration between RUF and PUF was evident probably due to the exclusion effect of proteins (de Man, 1962). During the first nanofiltration, the remaining proteins were retained and about 30\% of sugars pass the membrane (Table 1; Figure 1). The final nanofiltration permitted the complete recovery of the remaining sugars, whereas the final permeate was mainly composed of water and milk soluble salts (Table 1; Figure 1), which agreed with Brans et al. (2004).

The great relative standard deviation (RSD) for SNF $(\mathrm{RSD}=74 \%)$, sugars $(\mathrm{RSD}=120 \%)$, and protein $(\mathrm{RSD}=179 \%)$ of the whole data set can lead to better 


\section{Ultrafiltration}

\section{Proteins \\ Sugars}

Salts

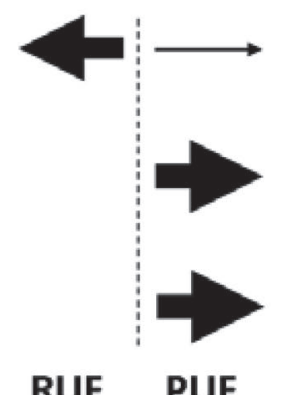

RUF PUF

\section{Nanofiltration 1}

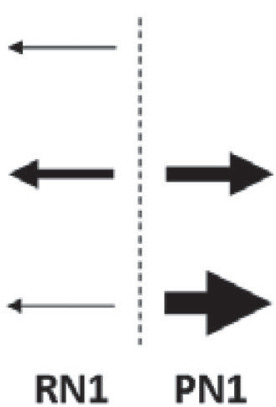

Nanofiltration 2

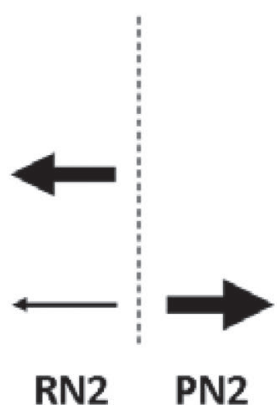

Figure 1. Scheme of the major constituents partition during the ultra- and nanofiltration processes. The thickness of arrows is proportional to constituent partition. RUF $=$ retentate ultrafiltered; $\mathrm{PUF}=$ permeate ultrafiltered; RN1 = retentate nanofiltered 1 ; PN1 = permeate nanofiltered $1 ; \mathrm{RN} 2=$ retentate nanofiltered $2 ; \mathrm{PN} 2=$ permeate nanofiltered 2.

identification of fingerprint and correction wavelengths performing PLS (Roggo et al., 2007; De Marchi et al., 2014). When considering the single fractions, RUF and RN1 showed the greater RSD for protein (14\% and $25 \%$, respectively), due to the variability in UF efficacy. On the other hand, PN1, RN2, and PN2 showed the greatest RSD in sugar content (17, 15, and 38\%, respectively), compared with the other fractions, with their sugar content being strongly dependent on the efficiency of the first nanofiltration step.

Samples from the same filtration step, when subset was based on concentration level or on Mahalanobis distance, were assigned to the same calibration group. As it can be retrieved from Table 1, groups established on concentration levels included for protein group 1 fractions obtained after the first UF (PUF, RN1, PN1, RN2, and PN2), whereas group 2 included DFM and DLM, and group 3 included RUF fraction. For glucose and galactose, DFM, RUF, PUF, PN1, and PN2 were included in group 1, whereas RN1 and RN2 constituted group 2 and 3, respectively. The SNF group 1 was formed by fractions with physiological or lower major constituent values (DFM, DLM, PUF, PN1, PN2), whereas group 2 included RUF and RN1, and group 3 included RN2 fraction. Even if grouping based on reference values can be easily set up, it should be done for each trait. From a practical point of view, having different groups for each component is more demanding in terms of resampling and maintenance. Figure 2 represented the fractions included in each cluster. Because clustering was based on the spectrum, the cluster was the same for all of the studied traits, facilitating the development of calibrations models and, eventually, the collection of new samples for too small subsets.

\section{FT-MIR Prediction Models}

As observed in Table 2, the RPD for all the proposed approaches was greater than 5 with better performances for protein and SNF content compared with glucose and galactose. Depending on the approach used to develop the prediction model, RPD for protein ranged from

Table 1. Descriptive statistics of collected samples

\begin{tabular}{|c|c|c|c|c|c|c|c|c|c|c|c|c|}
\hline $\begin{array}{l}\text { Milk } \\
\text { fraction }^{1}\end{array}$ & \multicolumn{3}{|c|}{ Glucose } & \multicolumn{3}{|c|}{ Galactose } & \multicolumn{3}{|c|}{ Protein } & \multicolumn{3}{|c|}{ SNF } \\
\hline DFM & 17 & $<0.005$ & $\mathrm{NC}^{2}$ & 17 & $<0.005$ & $\mathrm{NC}$ & 5 & 3.20 & 0.04 & 5 & 8.96 & 0.04 \\
\hline RUF & 18 & 2.08 & 0.20 & 18 & 2.11 & 0.19 & 15 & 12.98 & 1.81 & 15 & 19.17 & 2.20 \\
\hline PUF & 15 & 2.60 & 0.08 & 15 & 2.58 & 0.07 & 12 & 0.20 & 0.01 & 12 & 6.01 & 0.09 \\
\hline RN1 & 18 & 4.85 & 0.78 & 18 & 5.13 & 1.07 & 15 & 0.64 & 0.16 & 15 & 13.55 & 2.70 \\
\hline Total & 149 & 3.67 & 4.39 & 149 & 3.70 & 4.40 & 112 & 2.43 & 4.35 & 112 & 11.40 & 8.46 \\
\hline
\end{tabular}

${ }^{1} \mathrm{DFM}=$ defatted milk; DLM = delactosated milk; RUF = retentate ultrafiltered; PUF = permeate ultrafiltered; RN1 = retentate nanofiltered $1 ; \mathrm{PN} 1=$ permeate nanofiltered $1 ; \mathrm{RN} 2=$ retentate nanofiltered $2 ; \mathrm{PN} 2=$ permeate nanofiltered 2.

${ }^{2} \mathrm{NC}=$ not calculable. 
27.42 to 45.94 , for SNF from 17.80 to 18.48 , for galactose from 8.70 to 14.02 , and for glucose from 7.58 to 13.40. For glucose, galactose, and proteins, both RPD and $\mathrm{R}_{\mathrm{CV}}^{2}$ were improved when samples were grouped, probably because level and cluster approaches lead to a reduction of their SECV as indicated by Isaksson and Næs (1990) and Shenk and Westerhaus (1991). For glucose, galactose, and protein, SECV were reduced between 57 and $67 \%$ compared with the calibration developed on the whole data set. For sugars, the greatest RPD were obtained with the cluster approach, and for protein with the level approach. On the other hand, level and cluster approaches did not significantly improve SNF calibration, which could be explained by a slight increase of the SECV (Shenk and Westerhaus, 1991). Nevertheless, RPD values could be biased by the very high SD of the samples (Table 1), as reported by Bellon-Maurel et al. (2010) for soil samples.

Thus, the same statistics were also calculated considering only the results within each fractionation step, which decreased the SD (Table 1) and normal distribution was ensured, reporting the RPD in Table 3. Moreover, statistical analysis within each fractionation

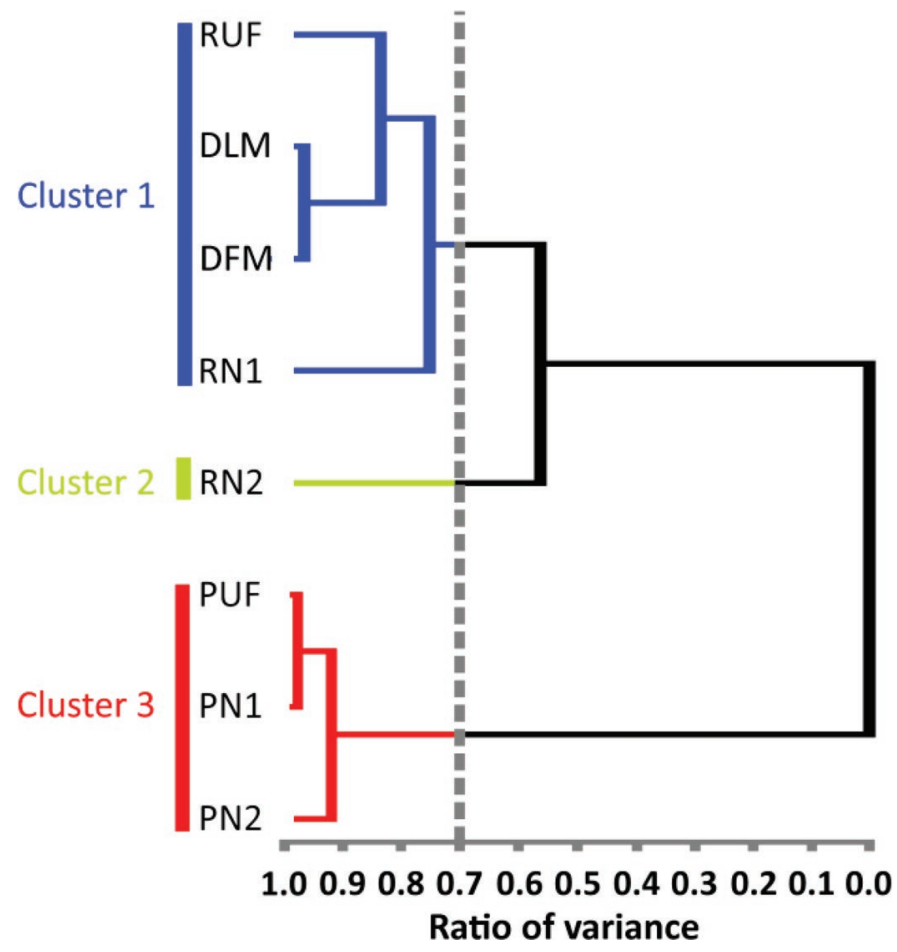

Figure 2. Result of the spectral clustering of the fractions. DFM $=$ defatted milk; DLM $=$ delactosated milk; RUF $=$ retentate ultrafiltered: $\mathrm{PUF}=$ permeate ultrafiltered; $\mathrm{RN} 1=$ retentate nanofiltered 1 ; PN1 = permeate nanofiltered $1 ; \mathrm{RN} 2=$ retentate nanofiltered 2; PN2 $=$ permeate nanofiltered 2 . The dashed line represents the threshold for cluster definition. The $\mathrm{x}$-axis shows the ratio of variance represented by the tree nodes. Color version available online.

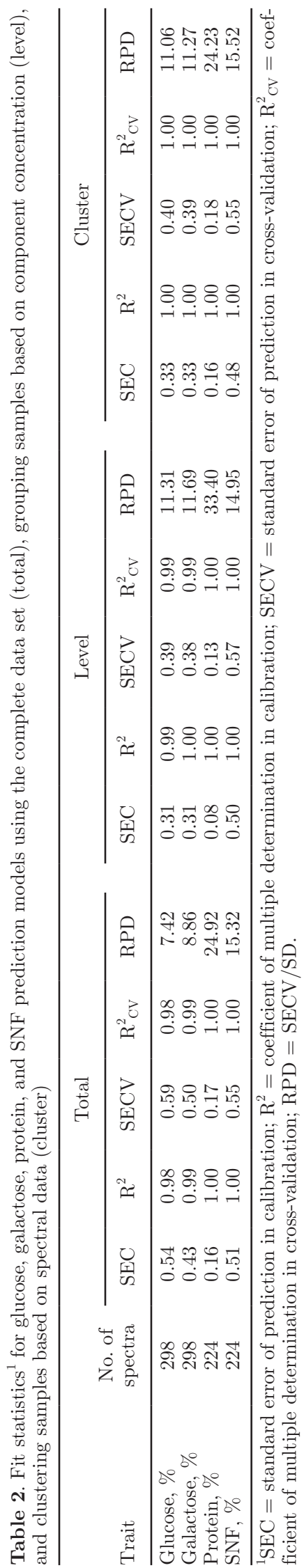

Journal of Dairy Science Vol. 101 No. 8, 2018 
step allowed us to verify the feasibility of the developed prediction models for at-line controls during processing. For sugars, RPD in the DFM fraction could not be calculated due to the concentration under the limit of detection $(0.005 \%$; Table 1$)$. Some of the calculated RPD included less than 60 spectra (Table 3), which could lead to an uncertain prediction, even if, for specific cases, 20 samples have been demonstrated to be sufficient for reliable calibrations (Isaksson and Næs, 1990; Hair et al., 2009). The RPD for each trait within a filtrated fraction (Table 3 ) decreased compared with the RPD considering all the filtration fraction together (Table 2). Level and cluster approaches improve the accuracy of the prediction model within each milk filtrated fraction (Table 3), as it was also observed considering all the fractions together (Table 2). Between those 2 approaches, level or cluster approach was the best one depending on the filtrated fraction and the trait (Table 3). For example, considering protein concentration, a level-based approach better performed for DFM, DLM, and RN1, whereas the cluster approach gave better results for RUF, PUF, PN1, RN2, and PN2 fractions (Table 3). The statistics obtained for each fraction compared with the whole data set calibration suggested that filtrated fractions with a greater concentration of the trait of interest resulted in better prediction models. Overall, RPD results demonstrated that the developed calibrations can be used for the quantification of components in almost all milk fractions, being more robust for protein content. Considering the single fractions, even if most RPD were between 2 and 3 , the obtained calibrations can be useful to highlight technical issues during the milk fractionation process (Sinnaeve et al., 1994; Williams, 2014).

\section{CONCLUSIONS}

Results of the present study showed that applying grouping protocols based on concentration level or spectra clustering increases the accuracy of the prediction model for protein and sugars in defatted, delactosated milk and its ultra- and nanofiltration stages. In terms of prediction equation maintenance, the possibility of having one equation that fits different fractions reduces the maintenance work compared with having one prediction equation for each fraction, and is a less expensive choice. In all 3 approaches (whole data set, level approach, and cluster approach), the best prediction models were obtained for protein, followed by SNF, whereas glucose and galactose performed similarly. Predictions decreased accuracy when RPD were calculated within each fraction, which could be related to the low number of samples within each fraction, the lower concentration of the traits in some fractions, and the limited variability of component concentrations within the fractions. Because none of the group approaches showed a superiority for all traits and fractions, the representativeness of the samples forming each group could be the main indicator for the final choice. Because most of the calculated RPD within each fraction and trait were between 2 and 3, further studies are needed to assess the feasibility of those models to finely monitor the filtration process.

\section{ACKNOWLEDGMENTS}

The authors thank Paolo Galvan and Dario Bianchi (Foss Italia) for his precious help and Luca Grigoletto (University of Padova, Italy) for his technical support.

Table 3. Ratio performance deviation in cross-validation (RPD) calculated within each fraction using the predicted values obtained with the prediction models following the 3 approaches $($ Tot $=$ whole database calibration; Lev $=$ reference data subset calibration; Clu $=$ cluster subset calibration)

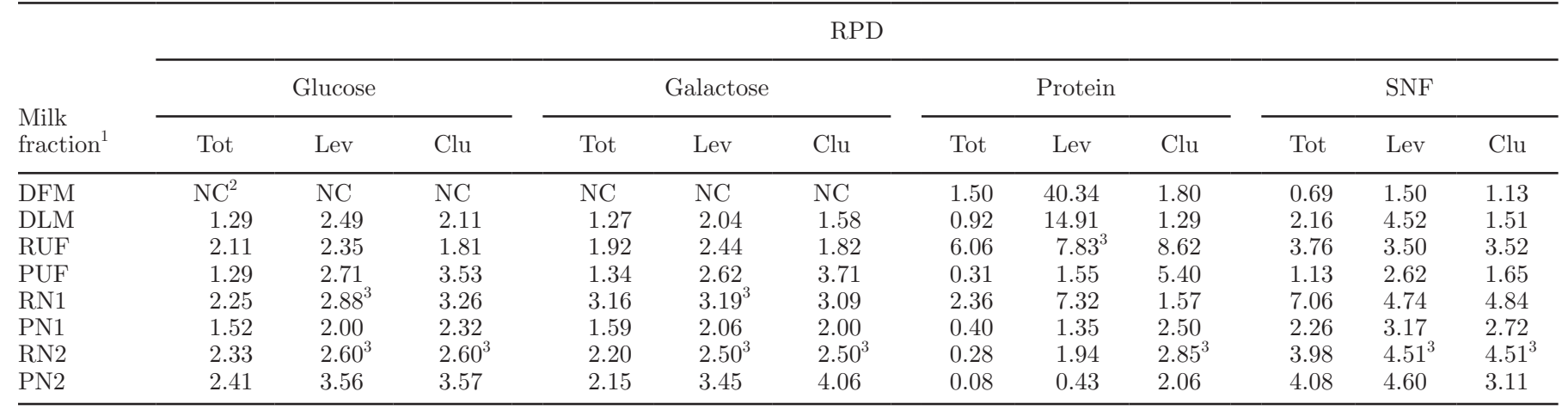

${ }^{1} \mathrm{DFM}=$ defatted milk; DLM $=$ delactosated milk, RUF $=$ retentate ultrafiltered, PUF $=$ permeate ultrafiltered, RN1 = retentate nanofiltered $1, \mathrm{PN} 1=$ permeate nanofiltered $1, \mathrm{RN} 2=$ retentate nanofiltered $2, \mathrm{PN} 2=$ permeate nanofiltered 2.

${ }^{2} \mathrm{NC}=$ not calculable.

${ }^{3}$ Calculated with less than 60 spectra. 
Granarolo Spa (Bologna, Italy) and the University of Padova (Padova, Italy) granted financial support for the project.

\section{REFERENCES}

Agarwal, S., R. L. W. Beausire, S. Patel, and H. Patel. 2015. Innovative uses of milk protein concentrates in product development. J. Food Sci. 80(Suppl 1):A23-A29. https://doi.org/10.1111/1750 $-3841.12807$.

Agelet, L. E., and C. R. Hurburgh. 2010. A tutorial on near infrared spectroscopy and its calibration. Crit. Rev. Anal. Chem. 40:246260. https://doi.org/10.1080/10408347.2010.515468.

Allen, D. M. 1974. The relationship between variable selection and data augmentation and a method for prediction. Technometrics 16:125-127. https://doi.org/10.2307/1267500.

AOAC International. 2000. Official Methods of Analysis. 17th ed. AOAC International, Gaithersburg, MD.

Barbano, D. M., and M. E. Dellavalle. 1987. Rapid method for determination of milk casein content by infrared analysis. J. Dairy Sci. 70:1524-1528. https://doi.org/10.3168/jds.S0022-0302(87)80179 -0 .

Bellon-Maurel, V., E. Fernandez-Ahumada, B. Palagos, J.-M. Roger, and A. McBratney. 2010. Critical review of chemometric indicators commonly used for assessing the quality of the prediction of soil attributes by NIR spectroscopy. Trends Analyt. Chem. 29:10731081. https://doi.org/10.1016/j.trac.2010.05.006.

Benedet, A., C. L. Manuelian, M. Penasa, M. Cassandro, F. Righi, M. Sternieri, P. Galimberti, A. V. Zambrini, and M. D. Marchi. 2018. Factors associated with herd bulk milk composition and technological traits in the Italian dairy industry. J. Dairy Sci. 101:934-943. https://doi.org/10.3168/jds.2017-12717.

Brans, G., C. G. P. H. Schroën, R. G. M. van der Sman, and R. M. Boom. 2004. Membrane fractionation of milk: State of the art and challenges. J. Membr. Sci. 243:263-272. https://doi.org/10.1016/j .memsci.2004.06.029.

De Marchi, M., V. Toffanin, M. Cassandro, and M. Penasa. 2014. Invited review: Mid-infrared spectroscopy as phenotyping tool for milk traits. J. Dairy Sci. 97:1171-1186. https://doi.org/10.3168/ jds.2013-6799.

de Man, J. M. 1962. Measurement of the partition of some milk constituents between the dissolved and colloidal phases. J. Dairy Res. 29:279-283. https://doi.org/10.1017/S0022029900011092.

Goulden, J. D. S. 1956. Infra-red spectroscopy of dairy products. J. Sci. Food Agric. 7:609-613. https://doi.org/10.1002/jsfa.2740070908.

Hair, J., W. Black, B. Babin, and R. Anderson. 2009. Multivariate Data Analysis. 7th ed. Prentice Hall.

Indyk, H. E., M. J. Edwards, and D. C. Woollard. 1996. High performance liquid chromatographic analysis of lactose-hydrolysed milk. Food Chem. 57:575-580. https://doi.org/10.1016/S0308 -8146(96)00195-1.

Isaksson, T., and T. Næs. 1990. Selection of samples for calibration in near-infrared spectroscopy. Part II: Selection based on spectral measurements. Appl. Spectrosc. 44:1152-1158.

Khatkar, S. K., V. K. Gupta, and A. B. Khatkar. 2014. Studies on preparation of medium fat liquid dairy whitener from buffalo milk employing ultrafiltration process. J. Food Sci. Technol. 51:19561964. https://doi.org/10.1007/s13197-014-1259-0.

Kumar, P., N. Sharma, R. Ranjan, S. Kumar, Z. F. Bhat, and D. K. Jeong. 2013. Perspective of membrane technology in dairy industry: A review. Asian-australas. J. Anim. Sci. 26:1347-1358. https://doi.org/10.5713/ajas.2013.13082.
McDermott, A., M. D. Marchi, D. P. Berry, G. Visentin, M. A. Fenelon, N. Lopez-Villalobos, and S. McParland. 2017. Cow and environmental factors associated with protein fractions and free amino acids predicted using mid-infrared spectroscopy in bovine milk. J. Dairy Sci. 100:6272-6284. https://doi.org/10.3168/jds.2016-12410.

McKenna, D. 2001. Measuring moisture in cheese by near infrared absorption spectroscopy. J. AOAC Int. 84:623-628.

Moreno-Montoro, M., M. Olalla, R. Giménez-Martínez, T. BergillosMeca, M. D. Ruiz-López, C. Cabrera-Vique, R. Artacho, and M. Navarro-Alarcón. 2015. Ultrafiltration of skimmed goat milk increases its nutritional value by concentrating nonfat solids such as proteins, Ca, P, Mg, and Zn. J. Dairy Sci. 98:7628-7634. https:// doi.org/10.3168/jds.2015-9939.

Naes, T., T. Isaksson, T. Fearn, and T. Davies. 2002. A User Friendly Guide to Multivariate Calibration and Classification. NIR Publications, Chichester, UK.

Pouliot, Y. 2008. Membrane processes in dairy technology-From a simple idea to worldwide panacea. Int. Dairy J. 18:735-740. https://doi.org/10.1016/j.idairyj.2008.03.005.

Roggo, Y., P. Chalus, L. Maurer, C. Lema-Martinez, A. Edmond, and N. Jent. 2007. A review of near infrared spectroscopy and chemometrics in pharmaceutical technologies. J. Pharm. Biomed. Anal. 44:683-700. https://doi.org/10.1016/j.jpba.2007.03.023.

Shenk, J. S., and M. O. Westerhaus. 1991. Population definition, sample selection, and calibration procedures for near infrared reflectance spectroscopy. Crop Sci. 31:469-474. https://doi.org/10 $.2135 /$ cropsci1991.0011183X003100020049x.

Sinnaeve, G., P. Dardenne, R. Agneessens, and R. Biston. 1994. The use of near infrared spectroscopy for the analysis of fresh grass silage. J. Near Infrared Spectrosc. 2:79-84. https://doi.org/10.1255/ jnirs.34.

Sokal, R. R., and C. D. Michener. 1958. A statistical method for evaluating systematic relationships. Univ. Kans. Sci. Bull. 38:1409-1438.

Solís-Oba, M., O. Teniza-García, M. Rojas-López, R. Delgado-Macuil, J. Díaz-Reyes, and R. Ruiz. 2011. Application of infrared spectroscopy to the monitoring of lactose and protein from whey after ultra and nano filtration process. J. Mex. Chem. Soc. 55:190-193.

Sturaro, A., M. Penasa, M. Cassandro, A. Varotto, and M. D. Marchi. 2014. Effect of microparticulated whey proteins on milk coagulation properties. J. Dairy Sci. 97:6729-6736. https://doi.org/10 $.3168 /$ jds.2014-8157.

Visentin, G., M. De Marchi, D. P. Berry, A. McDermott, M. A. Fenelon, M. Penasa, and S. McParland. 2017. Factors associated with milk processing characteristics predicted by mid-infrared spectroscopy in a large database of dairy cows. J. Dairy Sci. 100:32933304. https://doi.org/10.3168/jds.2016-12028.

Visentin, G., M. Penasa, P. Gottardo, M. Cassandro, and M. De Marchi. 2016. Predictive ability of mid-infrared spectroscopy for major mineral composition and coagulation traits of bovine milk by using the uninformative variable selection algorithm. J. Dairy Sci. 99:8137-8145. https://doi.org/10.3168/jds.2016-11053.

Wang, L., D.-W. Sun, H. Pu, and J.-H. Cheng. 2017. Quality analysis, classification, and authentication of liquid foods by near-infrared spectroscopy: A review of recent research developments. Crit. Rev. Food Sci. Nutr. 57:1524-1538. https://doi.org/10.1080/10408398 .2015 .1115954 .

Williams, P. 2014. The RPD statistic: A tutorial note. NIR News 25:22-26. https://doi.org/10.1255/nirn.1419.

Williams, P., and D. Sobering. 1993. Comparison of commercial near infrared transmittance and reflectance instruments for analysis of whole grains and seeds. J. Infrared Spectrosc. 1:25. https://doi .org/10.1255/jnirs.3. 